Accelerator mass spectrometry of Strontium-90 for homeland security, environmental monitoring, and human health

S. J. Tumey, T. A. Brown, T. F. Hamilton, D. J. Hillegonds

March 19, 2008

Nuclear Instruments and Methods in Physics Research B: Beam Interactions with Material and Atoms 
This document was prepared as an account of work sponsored by an agency of the United States government. Neither the United States government nor Lawrence Livermore National Security, LLC, nor any of their employees makes any warranty, expressed or implied, or assumes any legal liability or responsibility for the accuracy, completeness, or usefulness of any information, apparatus, product, or process disclosed, or represents that its use would not infringe privately owned rights. Reference herein to any specific commercial product, process, or service by trade name, trademark, manufacturer, or otherwise does not necessarily constitute or imply its endorsement, recommendation, or favoring by the United States government or Lawrence Livermore National Security, LLC. The views and opinions of authors expressed herein do not necessarily state or reflect those of the United States government or Lawrence Livermore National Security, LLC, and shall not be used for advertising or product endorsement purposes. 


\section{Accelerator mass spectrometry of Strontium-90 for homeland security, environmental monitoring, and human health}

Scott J. Tumey ${ }^{1, *}$, Thomas A. Brown ${ }^{1}$, Terry E. Hamilton ${ }^{1}$, Darren J. Hillegonds ${ }^{1}$

1 - Center for Accelerator Mass Spectrometry, Lawrence Livermore National

Laboratory, 7000 East Avenue, Livermore, CA 94551, USA

PACS: 89.20.-a; 29.30.-h; 07.88.+y

Keywords: AMS, Sr-90, Environmental monitoring

\section{Abstract}

Strontium-90 is one of the most hazardous materials managed by agencies charged with protecting the public from radiation. Traditional radiometric methods have been limited by low sample throughput and slow turnaround times. Mass spectrometry offers the advantage of shorter analysis times and the ability to measure samples immediately after processing, however conventional mass spectrometric techniques are susceptible to molecular isobaric interferences that limit their overall sensitivity. In contrast, accelerator mass spectrometry is insensitive to molecular interferences and we have therefore begun developing a method for determination of ${ }^{90} \mathrm{Sr}$ by accelerator mass

\footnotetext{
* Corresponding author: 7000 East Avenue, L-397; Livermore, CA 94551; +1-925-4239012; tumey2@1lnl.gov
} 
spectrometry. Despite a pervasive interference from ${ }^{90} \mathrm{Zr}$, our initial development has yielded an instrumental background of $\sim 10^{8}$ atoms $(75 \mathrm{mBq})$ per sample. Further refinement of our system (e.g., redesign of our detector, use of alternative target materials) is expected to push the background below $10^{6}$ atoms, close to the theoretical limit for AMS. Once we have refined our system and developed suitable sample preparation protocols, we will utilize our capability in applications to homeland security, environmental monitoring, and human health.

\section{Introduction}

Strontium-90 is one of the most hazardous constituents of nuclear waste because it is produced in high yield ( $4 \%)$ by fission of uranium and plutonium, and its half-life (28.78 a) is such that in addition to having a relatively high specific activity, it will also persist in the environment for times relevant to humans. As a member of the alkaline earth elements, strontium has similar chemical properties to calcium and is therefore a prolific bone seeker. Furthermore, its ionic nature allows great mobility in the environment. These reasons, coupled with the historical link between ${ }^{90} \mathrm{Sr}$ exposure and diseases such as leukemia, make it one of the most important substances managed by agencies charged with protecting the public from radiation such as the United States Department of Energy and the International Atomic Energy Agency.

Determination of ${ }^{90} \mathrm{Sr}$ by traditional radiometric methods is problematic due to its broad, featureless beta decay spectrum. The preferred method has traditionally been 
measurement of the more easily detected ${ }^{90} \mathrm{Y}$ daughter [1-3] and can attain a limit of detection of several $\mathrm{mBq}$. This approach has a couple of key limitations. First, over two weeks are required following radiochemical separation in order to establish secular equilibrium. Furthermore, measurement times are very long (hours or even days per sample), especially for low-level samples. These two factors limit both the turnaround time and overall sample throughput attainable by decay counting.

Recently, mass spectrometric methods have been investigated as an alternative for the determination of ${ }^{90} \mathrm{Sr}$ [4-5]. Mass spectrometry, which measures radioisotopes directly rather than the by-products of their decay, offers the advantages of more immediate and rapid analysis (minutes per sample) which enable quicker turnaround time and greater sample throughput. However traditional mass spectrometric methods have been limited by molecular isobaric interferences. In contrast, accelerator mass spectrometry (AMS) is insensitive to molecular interferences, and is routinely used to quantify long-lived radioisotopes in samples containing as few as $10^{5}$ atoms in the presence of an overwhelming stable isotope (i.e., isotope ratios $<10^{-15}$ ) [6-7]. For ${ }^{90} \mathrm{Sr}$ this would translate into a detection limit of less than $0.1 \mathrm{mBq}$, however interference from ${ }^{90} \mathrm{Zr}$ makes achieving this theoretical limit challenging, as discussed below.

The benefits of AMS are well suited to the determination of ${ }^{90} \mathrm{Sr}$ for a wide variety of applications. In the emergency response to a radiological incident (e.g., an accidental release from a power plant) a large number of sample measurements would be required with quick turnaround in order to assess the exposure of the population to ${ }^{90} \mathrm{Sr}$, 
and to evaluate the extent of contamination to surrounding areas. Environmental monitoring programs would also benefit from a ${ }^{90} \mathrm{Sr}$ AMS method. The US Marshall Islands Program has identified ${ }^{90} \mathrm{Sr}$ as a high priority isotope of interest, but has yet to incorporate it into its routine measurement protocols because of the sensitivity and throughput limitations of existing methods. $\mathrm{A}^{90} \mathrm{Sr}$ AMS would also have an important application to human health studies. For example, the drug strontium ranelate has recently shown efficacy in the treatment of osteoporosis [8-9] however, its exact functionality is under debate [10-11]. The enhanced sensitivity of AMS would allow ${ }^{90} \mathrm{Sr}$ to be used a biokinetic tracer to elucidate the functionality of strontium ranelate which would have great impact on the development of future bone related disease treatments.

\section{Experimental}

Based upon the encouraging experience of other AMS labs [12-14], we have begun development of ${ }^{90} \mathrm{Sr}$ measurement capabilities at the Lawrence Livermore National Laboratory (LLNL) Center for AMS (CAMS). This development has been performed using the CAMS heavy-isotope system, which is described elsewhere [15].

\section{Results and Discussion}

The development of an AMS method for a new isotope can be challenging because there are a number of inter-related parameters that must be iteratively determined. The first major decision is which material is most suitable to use as a target 
material in the ion source. Fortunately, the work of Middleton [16] makes this task much easier. As is the case with the other alkaline earth elements, elemental strontium is a poor negative ion producer, and the hydride is the preferred choice for maximizing negative ion yield. However, the hydride is produced by a time intensive reduction procedure and decomposes rapidly in air. By contrast, strontium fluoride precipitates easily from solution, is stable in air, and we were able to extract useful beam currents ( $\sim 500 \mathrm{nA}$ of $\mathrm{SrF}_{3}{ }^{-}$) from our ion source. One disadvantage to $\mathrm{SrF}_{2}$ is the lower fractional energy at the terminal which results in greater Coulomb explosion and less populated high charge states. Since one of the primary advantages of an AMS method for ${ }^{90} \mathrm{Sr}$ is high sample throughput and quick turnaround time, we have selected $\mathrm{SrF}_{2}$ as our target material.

The next important parameters to be determined are the appropriate terminal voltage to apply to the accelerator, and the optimum charge state to select from the distribution that exits the accelerator. This process is guided by examining the stripping yields for the isotope of interest. Since little experimental data have been published for Sr, we first calculated the energy dependent stripping yields based on the formalism developed by Sayer [17], and then confirmed these calculations experimentally as illustrated in Figure 1. We were constrained to gas stripping because foil stripping produced a beam that was too divergent to efficiently transport to the detector. If we were to use $\mathrm{SrH}_{2}$ instead of $\mathrm{SrF}_{2}$ as a target material we might be able to use foil stripping, however, the increase in sample preparation time associated with $\mathrm{SrH}_{2}$ was deemed unacceptable for our intended applications. We wanted to minimize the possibility of ions with equivalent mass to charge ratios that could potentially interfere 
with our measurement of ${ }^{90} \mathrm{Sr}$, so we selected the $7+$ charge state. Recent refurbishment of our accelerator tubes has limited the maximum terminal voltage to $8.3 \mathrm{MV}$, but in the future we plan to run at a higher voltage $(9.75 \mathrm{MV})$ to maximize both ion energy, and thus isobaric discrimination (discussed below), and stripping yield.

The standard detector in the CAMS heavy-isotope system is a two-anode gas ionization chamber with a $1.7 \mu \mathrm{m}$ aluminized Mylar entrance window. Preliminary measurements, which are shown in Fig. 2, demonstrate that the resolution of this detector is insufficient to fully resolve ${ }^{90} \mathrm{Sr}$ from the isobaric interferant ${ }^{90} \mathrm{Zr}$. In order to mitigate the impact of this interferant, we have developed two improvements to our measurement system. First, we have replaced the Mylar entrance window with a silicon nitride membrane. These membranes were first developed for AMS applications by the group at ETH-Zurich [18], and greatly enhance the resolving power of a gas ionization chamber. We are further improving the design of our detector by adding a third anode and optimizing the geometry of the anodes in order to maximize the differential energy loss between ${ }^{90} \mathrm{Sr}$ and ${ }^{90} \mathrm{Zr}$. By implementing these two features we anticipate at least a factor of ten enhancement of the resolving power of our detector.

The second improvement to our system came from the discovery that most of the

${ }^{90} \mathrm{Zr}$ interferant was originating from our standard aluminum target holders rather than the sample material itself. To solve this problem we experimented with alternative materials and discovered that high-purity nickel was an ideal replacement for aluminum. Nickel is commercially purified by a carbonyl extraction procedure that has high rejection of 
zirconium. By using nickel instead of aluminum for our target holders we achieved approximately a factor of ten suppression of ${ }^{90} \mathrm{Zr}$, as illustrated in Fig. 3. Another advantage is that nickel seems to enhance the negative ion yield of $\mathrm{SrF}_{3}^{-}$by a factor of two, thus enabling us to get twice as much ${ }^{90} \mathrm{Sr}$ beam into our detector. This effect was unexpected since $\mathrm{Ni}$ is more electronegative than $\mathrm{Al}$ (electron affinity of $1.156 \mathrm{eV}$ for $\mathrm{Ni}$ versus $0.433 \mathrm{eV}$ for $\mathrm{Al}$ ) and requires more thorough investigation to understand its cause.

\section{Conclusions and Future Work}

Our initial measurements of ${ }^{90} \mathrm{Sr}$ by AMS are encouraging. Our instrumental background typically ranges from $1 \times 10^{-11}$ to $5 \times 10^{-11}$ and is dominated by isobaric interference from ${ }^{90} \mathrm{Zr}$ intrinsic to our sample holders. Based on the mass of sample we load into our targets (equivalent to $1 \mathrm{mg} \mathrm{Sr}$ ), this background corresponds to $\sim 10^{8}$ atoms $(75 \mathrm{mBq})$ which is three orders of magnitude higher than the theoretical limit of AMS predicted by the overall efficiency (i.e., ionization, transmission, stripping) of our system. We have undertaken two strategies for improving our background. First, we are redesigning our detector to improve its ability to discriminate between ${ }^{90} \mathrm{Sr}$ and ${ }^{90} \mathrm{Zr}$. Second, we have replaced our standard aluminum target holders with high-purity nickel,

which suppressed ${ }^{90} \mathrm{Zr}$ by about a factor of ten. With these two improvements, we hope to push our sensitivity down below $10^{6}$ atoms, close to the theoretical limit for AMS.

We have also begun developing sample preparation protocols for real samples. The biggest challenge to sample preparation is the separation of $\mathrm{Sr}$ from $\mathrm{Ca}$. While 
calcium does not pose a direct interference to the measurement of ${ }^{90} \mathrm{Sr}$, it does present a sample loading problem since we are constrained by the maximum quantity of material that we can pack into our sample holders. For example, $125 \mathrm{~mL}$ of seawater contains the nominal quantity of $\operatorname{Sr}(1 \mathrm{mg})$ that we load into our target holders. However, the mass of $\mathrm{Ca}$ in this volume of seawater ( $>50 \mathrm{mg}$ ) exceeds the capacity of our target holders. Therefore, it is necessary to effectively separate $\mathrm{Ca}$ from our samples prior to precipitation of $\mathrm{SrF}_{2}$. Once we have improved our AMS measurement system and developed appropriate sample preparation methods, we will begin to measure ${ }^{90} \mathrm{Sr}$ in real samples in support of US DOE programs such as the Marshall Islands Program.

\section{Acknowledgements}

This work was performed under the auspices of the U.S. Department of Energy by Lawrence Livermore National Laboratory in part under Contract W-7405-Eng-48 and in part under Contract DE-AC52-07NA27344.

\section{References}

[1] P. Froidevaux, J.-J. Geering, J.-F. Valley, J. Radioanal. Nucl. Chem. 254 (2002) 23.

[2] L. Popov, X. Hou, S.P. Nielsen, Y. Yu, R. Djingova, I. Kuleff. J. Radioanal. Nucl. Chem. 269 (2006) 161.

[3] J.M. Torres, J.F. Garcia, M. Llaurado, G. Rauret, Analyst 121 (1996) 1737.

[4] V.F. Taylor, R.D. Evans, R.J. Cornett, Anal. Bioanal. Chem. 387 (2007) 343. 
[5] M.V. Zoriy, P. Ostapczuk, L. Halicz, R. Hille, J.S. Becker, Int. J. Mass Spec. 242 (2005) 203.

[6] M. Suter, Nucl. Inst. Meth. Phys. Res. B 223-4 (2004) 139.

[7] W. Kutschera, Int. J. Mass Spec. 242 (2005) 145.

[8] E. Seeman, B. Vellas, C. Benhamou, J.P. Aquino, J. Semler, J.M. Kaufman, K.

Hoszowski, A.R. Varela, C. Fiore, K. Brixen, J.Y. Reginster, S. Boonen, J. Bone Min.

Res. 21 (2006) 1113.

[9] J.Y. Reginster, E. Seeman, M.C. De Vernejoul, S. Adami, J. Compston, C. Phenekos, J.P. Devogelaer, M. Diaz Curiel, A. Sawicki, S. Goemaere, O.H. Sorensen, D.

Felsenberg, P.J. Meunier, J. Clin. Endocrin. Metab. 90 (2005) 2816.

[10] P.J. Marie, Bone 40 (2007) S5.

[11] G.M. Blake, E.M. Lewieck, D.L. Kendler, I. Fogelman, J. Clin. Densito. 10 (2007) 113.

[12] F. Arslan, M. Behrendt, W. Ernst, E. Finckh, G. Greb, F. Gumbmann, M. Haller, S. Hofmann, R. Karschnick, M. Klein, W. Kretschmer, J. Mackiol, G. Morgenroth, C.

Pagels, M. Schleicher, Nucl. Inst. Meth. Phys. Res. B 92 (1992) 39.

[13] G. Korschinek, T. Faestermann, S. Kastel, K. Knie, H.J. Maier, J. Fernandez-Niello, M. Rothenberger, L. Zerle, Nucl. Inst. Meth. Phys. Res. B 92 (1994) 146.

[14] M. Paul, D. Berkovits, L.D. Cecil, H. Feldstein, A. Hershkowitz, Y. Kashiv, S. Vogt, Nucl. Inst. Meth. Phys. Res. B 123 (1997) 394.

[15] A.A. Marchetti, T.A. Brown, C.C. Cox, T.F. Hamilton, R.E. Martinelli, J. Radioanal. Nucl. Chem. 263 (2005) 483. 
[16] R. Middleton, A Negative Ion Cookbook, University of Pennsylvania Press,

Philadelphia, 1989.

[17] R.O. Sayer, Revue De Physique Appliquee 12 (1977) 1543.

[18] M. Döbeli, C. Kottler, M. Stocker, S. Weinmann, H.-A. Synal, M. Grajcar, M. Suter, Nucl. Inst. Meth. Phys. Res. B 219-220 (2004) 415. 
Figures

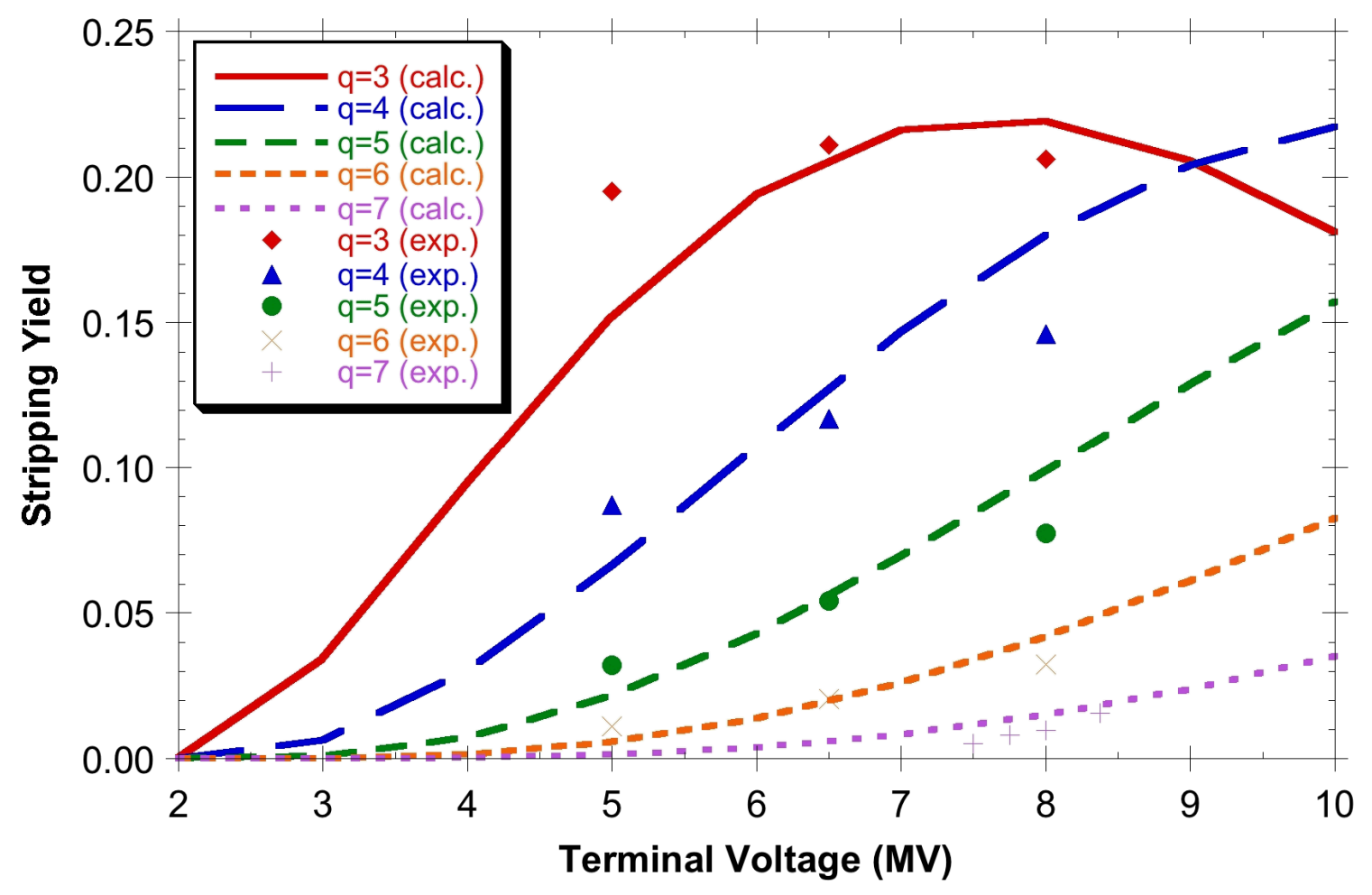

Figure 1. Calculated (lines) and experimental (symbols) values of stripping yield for various charge states of strontium $\left(\mathrm{SrF}_{2}^{-}\right.$injected into tandem at $\left.40 \mathrm{keV}\right)$ over a range of terminal voltages. The calculated values were determined using the formalism of Sayer $[17]$. 


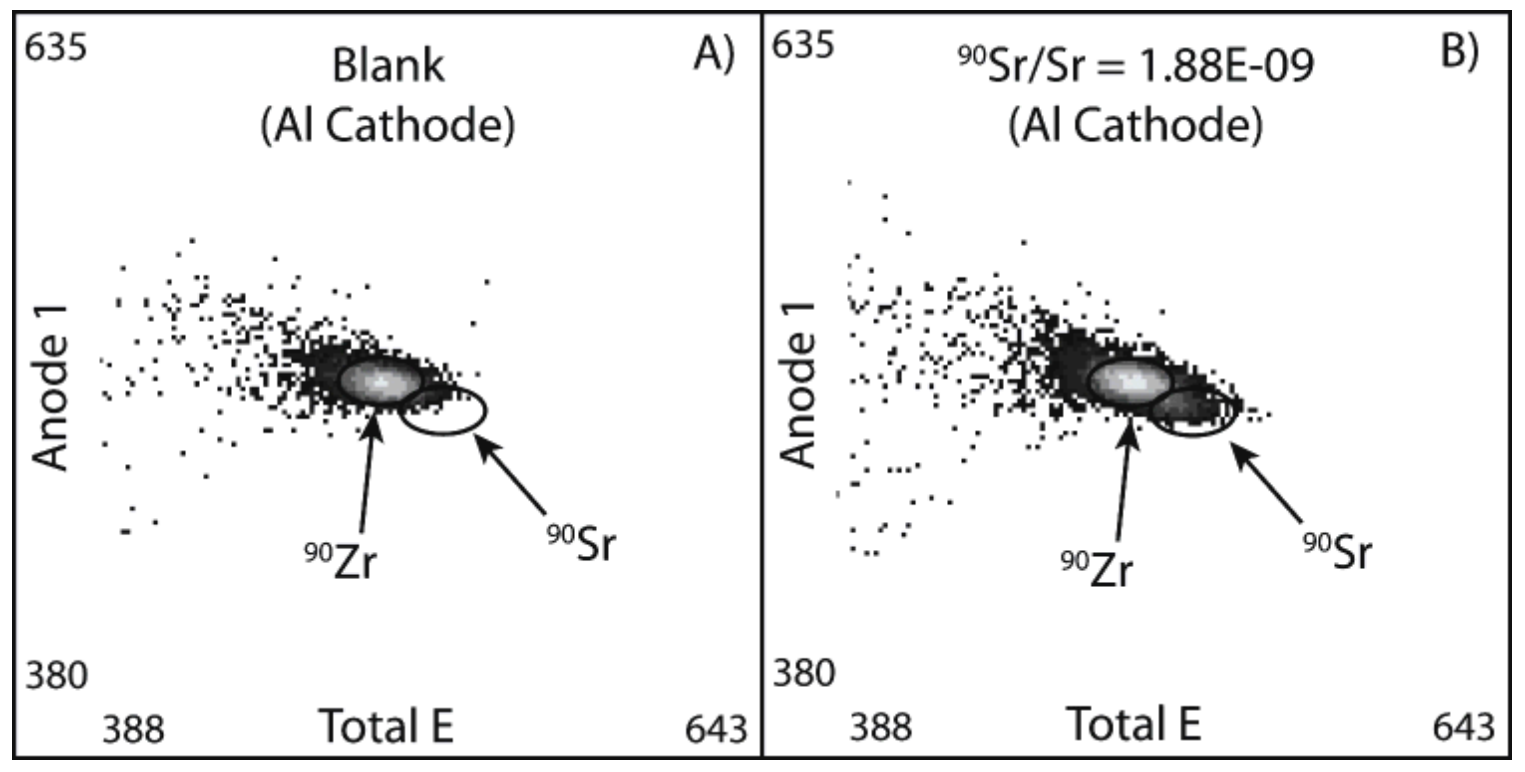

Figure 2. Two-dimensional energy loss spectra (Anode 1 vs. Total energy) obtained from A) a blank and B) a radiochemical standard $\left({ }^{90} \mathrm{Sr} / \mathrm{Sr}=1.88 \times 10^{-9}\right)$ loaded into our normal Al target holders and measured in the current heavy-isotope detector. Typical blank values from these targets range from $1 \times 10^{-11}$ to $5 \times 10^{-11}$. 


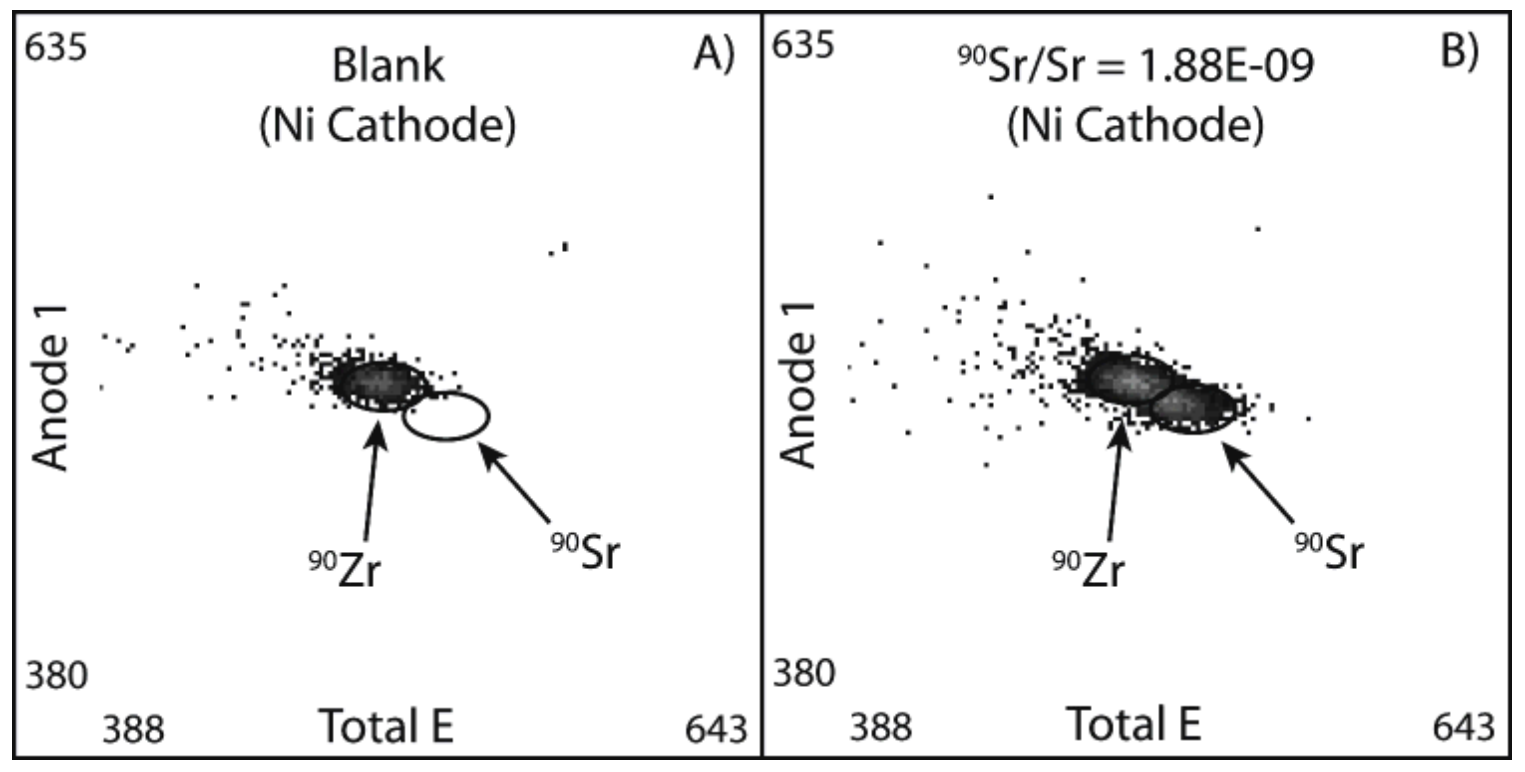

Figure 3. Two-dimensional energy loss spectra (Anode 1 vs. Total energy) obtained from A) a blank and B) a radiochemical standard $\left({ }^{90} \mathrm{Sr} / \mathrm{Sr}=1.88 \times 10^{-9}\right)$ loaded into modified target holders with high-purity nickel inserts and measured in the current heavy-isotope detector. These targets typically yield blank values approximately a factor of ten lower than our normal Al targets. 